\title{
Competencias Genéricas para la Educación en Odontolo- gía Forense: Pensamiento Crítico y Responsabilidad Social
}

\author{
Generic Skills for Education in Forensic Dentistry: Critical Thinking and Social \\ Responsibility
}

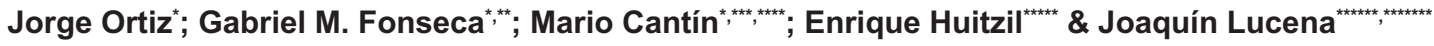

ORTIZ, J.; FONSECA, G. M.; CANTÍN, M.; HUITZIL, E. \& LUCENA, J. Competencias genéricas para la educación en odontología forense: Pensamiento crítico y responsabilidad social. Int. J. Odontostomat., 9(2):263-272, 2015.

RESUMEN: La Educación Basada en Competencias representa una estrategia de enseñanza-aprendizaje donde se busca que el estudiante logre un mejor desempeño en los entornos sociales cada vez más complejos a través del desarrollo de sus propias habilidades. Esto requiere que las instituciones educativas ofrezcan oportunidades curriculares que desplieguen Competencias Genéricas (CGs) transparentes y explícitas. Los desafíos contextuales de las ciencias forenses en general, y de la Odontología legal y forense en lo particular, han determinado una necesaria adaptación de sus contenidos conceptuales, procedimentales y actitudinales en la búsqueda de definición de sus campos específicos, desarrollo holístico de sus áreas de conocimiento, la adquisición de habilidades y experiencia, orientación hacia valores profesionales éticos, e integración de todos esos contenidos. Se presentan al Pensamiento Crítico y a la Responsabilidad Social como las CGs más idóneas para una educación focalizada en la Odontología legal y forense, como maneras de asumir el desafío de mejorar la calidad, consistencia y suficiencia de no sólo de futuros nuevos peritos sino también del profesional asistencial generalista, y se discute la trascendencia de formalizar estos aspectos según las realidades y paradigmas imperantes hoy en algunos países de habla hispana.

PALABRAS CLAVE: odontología forense, competencias genéricas, proceso de enseñanza-aprendizaje.

\section{INTRODUCCIÓN}

La sociedad contemporánea se encuentra en un rápido proceso de cambio, con necesidades de desempeño en entornos cada vez más complejos, problemas mal definidos, información contradictoria, colaboración informal y procesos dinámicos altamente integrados. EI concepto de competencia nace como una manera de desenvolverse exitosamente en tales contextos a través de la integración de conocimientos, habilidades y actitudes. La Educación Basada en Competencias (EBC) ha permitido a los estudiantes el capacitarse a sí mismos a través de una experiencia de aprendizaje auténtica y autodirigida (Wesselink et al., 2010).
Durante los últimos 20 años, las Competencias Genéricas (CGs), término acuñado para designar a cada una de esas habilidades o atributos, han representado un área de franco crecimiento en la educación superior. La clave de un rendimiento académico exitoso sustentado en el desarrollo efectivo de CGs radica en sus inclusiones en el proceso de enseñanza-aprendizaje (Murdoch-Eaton \& Whittle, 2012; El Tantawi et al., 2014). Sin embargo, dos preguntas han sido denotadas como relevantes para el desarrollo de la EBC: ¿qué competencias son necesarias para funcionar en un trabajo determinado?, y ¿de qué manera

\footnotetext{
Laboratorio de Pericias en Odontología Forense -LPO-, Centro de Investigación en Morfología Aplicada -CIMA-, Facultad de Odontología, Universidad de La Frontera, Temuco, Chile.

* Profesor Titular, Cátedra de Anatomía Patológica B, Facultad de Odontología, Universidad Nacional de Córdoba, Córdoba, Argentina.

*** Programa de Doctorado en Ciencias Morfológicas, Facultad de Medicina. Departamento Integral del Adulto, Facultad de Odontología, Universidad de La Frontera, Chile.

**** Centro de Investigación en Ciencias Biomédicas, Universidad Autónoma de Chile, Temuco, Chile.

Profesor Titular, Facultad de Estomatología, Benemérita Universidad Autónoma de Puebla, Puebla, México.

Profesor Asociado, Área de Medicina Forense, Universidad de Cádiz, Cádiz, España.

...uru* Servicio de Patología Forense, Instituto de Medicina Legal, Sevilla, España.

Financiamiento: Programa Atracción e Inserción de Capital Humano Avanzado MEC 80140078, CONICYT - Chile.
} 
esas competencias deberían ser desarrolladas? (Wesselink et al.).

Las ciencias forenses han contribuido con los tribunales de justicia suministrando la evidencia necesaria tanto para condenar criminales como para exonerar inocentes. En especial los últimos, los avances en determinadas disciplinas (especialmente las dedicadas a la genética) han demostrado un gran potencial al incrementar notablemente las posibilidades de identificar individuos superando a las sólo basadas en elementos subjetivos o valoraciones circunstanciales. Los esfuerzos de la comunidad científica forense se han enfocado en aumentar la certeza y confiabilidad de los métodos utilizados, el número de científicos calificados y el uso de buenas prácticas. Así, preparar nuevas generaciones de expertos (incluso desde el pregrado), formados de manera continua, formal y estructurada, abarcando además a la educación de los usuarios del análisis pericial (jueces, abogados, estudiantes de Derecho, instituciones y agencias gubernamentales), se han transformado en propósitos esenciales de una capacitación enfocada en comprender las bases científicas de las diferentes disciplinas forenses. La validez subyacente de las diferentes técnicas puede afectar significativamente la interpretación (y por ende, los resultados) de los hallazgos (Committee on Identifying the Needs of the Forensic Science Community et al., 2009).

Ciocca (2010), define a la Odontología Legal y Forense, como la "disciplina, ciencia o especialidad que en el ámbito médico-legal vincula al cirujano-dentista con la Ley, participando con los conocimientos de su incumbencia en la solución de problemas judiciales, y por otra parte colaborando en la gestación y el estudio del ordenamientojurídico que regula su ejercicio profesional habitual". Sin embargo, se ha referido que esta disciplina excede con creces los límites impuestos por la sola especialización. Algunos autores han establecido que, dado su básico (y comprometido) rol diagnóstico y responsable socialmente, existe la necesidad de implementar contenidos de odontología forense en el pregrado, en el postgrado a través de actividades de formación continua-, o incluso obligando a todo egresado a asistir a conferencias nacionales o internacionales de la disciplina (Avon, 2004; Singh et al., 2014).

Aunque la EBC constituye un desarrollo complejo que integra muchos ítems en la educación contemporánea, son escasos los estudios que logran incorporar todos sus aspectos más relevantes
(Wesselink et al.). En Temuco (Chile), la Universidad de La Frontera aprobó el año 2007 una Política de Formación Profesional cuya definición del Perfil del Titulado incorpora la declaración de propósitos con los que sus diferentes carreras inspiran sus diseños curriculares, estilos de docencia y evaluaciones. Este documento ha definido y adoptado una serie de CGs potenciadas por metodologías activas centradas en el estudiante y en su desarrollo, donde interactúan elementos cognitivos y motivacionales: Comunicación verbal y escrita en castellano; Comprensión lectora; Comunicación en inglés; Uso de las tecnologías de la información y comunicación; Aprender a aprender; Pensamiento crítico; Pensamiento complejo; Trabajo en equipo; Emprendimiento; Liderazgo y Responsabilidad Social (CIP, 2015).

Se presentan al Pensamiento Crítico y a la Responsabilidad Social como las CGs más idóneas para una educación focalizada en la disciplina Odontología Forense, como maneras de asumir el desafío de mejorar la calidad, consistencia y suficiencia de no sólo de futuros nuevos peritos sino también del profesional asistencial generalista, y se discute la trascendencia de formalizar estos aspectos según las realidades y paradigmas imperantes hoy en algunos países de habla hispana.

EBC y Ciencias Forenses. Los modelos educativos han sufrido diferentes modificaciones según el paradigma imperante y su enfoque biopsicosocial (Huitzil Muñoz, 2011; Bergsmann et al., 2015). Aunque su origen en los años ' 60 en los Estados Unidos no tuvo el éxito esperado, su renacimiento en la Europa de los '90 permitió una aproximación más holística, con competencias contextualizadas en componentes funcionales, conductuales, cognitivos y éticos (Wesselink et al.). Quizás uno de los elementos más favorables para la EBC ha sido la necesidad de integrar lo aprendido con la práctica, donde los estudiantes deben tener la oportunidad de experimentar diferentes contextos para poder crear nuevo conocimiento y práctica reflejando sus propias experiencias. Los modelos más fructíferos de aprendizaje han sido aquellos donde se alternan la teoría y la práctica, conectados a través de una tarea unificada de aprendizaje (Wesselink et al.).

Aunque las instituciones de educación superior desarrollan en la actualidad un cambio de currículos basados en contenidos, a basados en competencias y consecuentemente a una EBC (Bergsmann et al.), las instancias pretendiendo identificar las habilidades y competencias para la enseñanza superior de disci- 
plinas con una orientación forense son absolutamente escasas. Mason \& Phipps (2010), al entrevistar a auxiliares médicos en el Reino Unido, identificaron un grupo de competencias específicas en aquellos dedicados a labores forenses. Para los autores, esto posee un gran impacto en el desarrollo de los currículos y en las políticas de gestión de roles al respecto.

Jones (2009) ha establecido que la epistemología disciplinaria juega un papel importante en la construcción y enseñanza de las CGs; éstas y sus atributos pueden ser concebidos de manera diferente en cada disciplina según sus definiciones, importancia relativa o grado de integración en su contenido específico. Para el autor, las CGs constituyen más que un conjunto de habilidades que trascienden disciplinas, y deben ser entendidas como una práctica social. Esta concepción permitiría reformular la manera en que los educadores generan cierto tipo de comportamientos, junto con promover determinadas actitudes y prácticas en los estudiantes, a menudo de manera tácita.

Las disciplinas forenses en lo particular, proponen desafíos contextuales específicos: sus currículos deberían como mínimo asegurar a sus estudiantes: 1) Entender los tópicos esenciales en las disciplinas científicas forenses, incluyendo la reducción de los márgenes de error; 2) Desarrollar entendimiento de las áreas del conocimiento que son esenciales a la cien- cia forense; 3) Adquirir habilidades y experiencia en la aplicación de los conceptos científicos forenses básicos y de los específicos para la resolución de problemas; 4) El ser orientado en valores profesionales, conceptuales y éticos; y 5) Demostrar integración de conocimiento y habilidades mediante experiencias de cierre, así como herramientas formales y objetivas, exámenes integradores, tesis o proyectos de investigación (Committee on Identifying the Needs of the Forensic Science Community et al.).

CG Pensamiento Crítico. Siguiendo el documento UFRO (Tabla I), esta CG ha sido definida como la "habilidad para evaluar $\mathrm{o}$ analizar la estructura y consistencia de los razonamientos, particularmente opiniones o afirmaciones, escrutando las ideas, juicios o acciones ya sean propias o ajenas y los fundamentos de ellas, antes de aceptarlas como válidas". Sus elementos constituyentes están compuestos de: A) Análisis de la Realidad: La CG se relaciona con la actitud ante la realidad, la identificación y valoración de los hechos, opiniones y juicios en las argumentaciones; B) Elaboración de juicios: La CG se vincula al proceso de construcción de juicios y argumentos en base a un proceso de análisis reflexivo fundamentado; C) Habilidad de reflexión y análisis sobre las consecuencias y efectos de las decisiones, conclusiones e interpretaciones, en base a una manera de pensar que denota autonomía y compromiso intelectual.

Tabla I. Niveles de Dominio Competencia Genérica Pensamiento Crítico (UFRO).

\begin{tabular}{|c|c|c|}
\hline Nivel & Nivel de Dominio & Indicador conductual \\
\hline \multirow[t]{2}{*}{1} & \multirow[t]{2}{*}{$\begin{array}{l}\text { Se hace preguntas sobre la realidad que le } \\
\text { rodea, cuestiona y formula juicios. }\end{array}$} & $\begin{array}{l}\text { A.- Posee una actitud crítica ante la realidad en que está } \\
\text { inserto. }\end{array}$ \\
\hline & & $\begin{array}{l}\text { B.- Identifica los principios o ideas que sustentan un juicio. } \\
\text { C.- Emite juicios y valoraciones personales. }\end{array}$ \\
\hline \multirow[t]{2}{*}{2} & \multirow[t]{2}{*}{$\begin{array}{l}\text { Cuestiona la realidad, reconoce que los } \\
\text { juicios requieren fundamentación y valora } \\
\text { la opinión de otros. }\end{array}$} & $\begin{array}{l}\text { A.- Diferencia hechos objetivos de opiniones y valoraciones. } \\
\text { B.- Argumenta sus juicios desde modelos y valores que lo } \\
\text { sustentan. }\end{array}$ \\
\hline & & $\begin{array}{l}\text { C.- Formula juicios y valoraciones considerando los } \\
\text { argumentos de otros. }\end{array}$ \\
\hline \multirow[t]{3}{*}{3} & \multirow{3}{*}{$\begin{array}{l}\text { Analiza la coherencia de juicios propios y } \\
\text { ajenos, fundamentando de manera } \\
\text { pertinente sus decisiones y propuestas. }\end{array}$} & $\begin{array}{l}\text { A. - Formula preguntas y cuestiona la realidad, reflexionando } \\
\text { acerca de la misma. }\end{array}$ \\
\hline & & $\begin{array}{l}\text { B. - Fundamenta y argumenta juicios en base a criterios } \\
\text { apropiados, analizando su pertinencia y relevancia. }\end{array}$ \\
\hline & & $\begin{array}{l}\text { C.- Formula con fundamento sus propios juicios y } \\
\text { valoraciones incorporando de manera reflexiva juicios o } \\
\text { decisiones basadas en argumentos de otros. }\end{array}$ \\
\hline
\end{tabular}

Nivel 1: Competencia en la realización de una gama acotada de actividades, en su mayoría sencillas y de resultados predecibles; Nivel 2: Competencia en la realización de una gama mayor de actividades. El estudiante cuenta con un mínimo de autonomía, recibe un alto grado de supervisión y se desenvuelve de forma suficiente.

Nivel 3: Competencia en la realización de una gama variada de actividades, en diversos contextos. El estudiante actúa con mayor autonomía y asume la responsabilidad de su desempeño. 
Se ha considerado al pensamiento crítico como la raíz del proceso "educativo", y se postula que fomentar esta CG libera la mente, sus habilidades y atributos. La capacitación de los profesionales de la salud para reconocer sobreentendidos, valores o prejuicios, para evaluar evidencias, interpretar datos y explorar argumentos mediante mecanismos intelectuales de discriminación, precisión y juicio, es un desafío clave para los educadores médicos (Murdoch-Eaton \& Whittle). El pensamiento crítico puede ser aplicado en distintos contextos siguiendo como base el método científico, pero la manera en que éste se exprese podrá variar; a diferencia de lo que sucede en otras disciplinas, en el ámbito académico de las leyes debe ser entendido como un examen cuidadoso de la evidencia, de la lógica, de las suposiciones, del conocimiento del contexto social y del sentido de justicia. En medicina (más importante aún), el pensamiento crítico busca enfocarse en un buen razonamiento clínico, lo que requiere de un entendimiento de la medicina basada en evidencia y conciencia de cuestiones éticas. La odontología forense (por definición, la ciencia odontológica vinculada a la Ley), requiere de un profesional con un alto sentido ético que aplique los conocimientos basados en evidencia al servicio de los tribunales (Jones).

En el apartado referido a "Educación y Capacitación en Ciencias Forenses", la National Academy of Sciences de los Estados Unidos (NAS) especifica "...entre las diferentes habilidades, la educación en ciencias forenses debe brindar las herramientas necesarias para comprender las probabilidades y límites de tomar decisiones bajo condiciones de incertidumbre" (Committee on Identifying the Needs of the Forensic Science Community et al.). El controvertido caso Daubert vs. Merrel Dow Pharmaceuticals Inc. introdujo en las cortes federales norteamericanas (y en la comunidad científica mundial) nuevos estándares para la admisibilidad de los peritos y de sus técnicas; más allá de enmarcar su forma y función, estas normativas suponen delimitar a un experto conocedor en profundidad de un tema y con capacidad de análisis, una persona dotada de conocimientos y de opinión fundada, a la vez provista de experiencia (Fonseca, 2012).

Uno de los grandes hitos de la educación odontológica ha sido el potenciar las habilidades del estudiante para pensar críticamente y formar sus propias opiniones respecto de lo ofrecido por la literatura científica. Esta CG ha sido enfatizada por la American Dental Education Association (ADEA) como una com- petencia básica para posibilitar una odontología basada en evidencia (Lallier, 2014). El desarrollar habilidades para el pensamiento crítico parece ser una posible solución para el potencial experto odontólogo forense. La evaluación precisa, el discernimiento no sólo de los alcances sino también de las limitaciones de la propia experticia y prácticas asociadas, brindaría una conducta reflexiva, crítica y honesta, todas cualidades indispensables en el perito (Fonseca).

CG Responsabilidad Social. La UFRO ha definido esta CG como la "capacidad y obligación de responder ante la sociedad como un todo, por acciones $u$ omisiones y que se ejercen, cuando corresponde, desde algunas personas hacia las otras. Favorece la igualdad de oportunidades al permitir la manifestación de las potencialidades más allá de impedimentos estructurales de carácter económico, social, político o cultural" (Tabla II). Posee como elementos constituyentes: A) Responsabilidad ante sí mismo: Referido básicamente a la condición de responsabilidad propia y personal que debe tener un individuo consigo mismo. Considera el cuidado de sí mismo, la responsabilidad en sus acciones y el cumplimiento de propósitos personales, etc.; B) Responsabilidad ante los cercanos: Considera que toda acción y decisión propia tiene un efecto en los cercanos el cual resulta fundamental para la convivencia y el avance en las metas comunes de un equipo o colectivo; C) Responsabilidad ante el entorno: Se es responsable social y profesionalmente cuando se es consciente de cómo las acciones propias o de un colectivo afectan al entorno mayor y sobre este punto se requiere una acción pensada y reflexiva que evalúe consecuencias de los actos y decisiones.

El proyecto Tuning-America, dentro de sus 27 competencias genéricas, ha establecido a la responsabilidad social como la quinta en importancia asignada por las organizaciones de la sociedad civil. Al tomar las universidades el desafío de difundir y poner en práctica un conjunto de principios y valores generales y específicos a través de su quehacer, sus estudiantes asumen el foco como personas reflexivas capaces de ver las consecuencias de su pensar, sentir y hacer en el medio en que se vive. Esto pretende ser logrado incorporando en el currículo y perfiles de egreso de las carreras de pregrado la Responsabilidad Social como un comportamiento presente para que en el ejercicio profesional, los egresados contribuyan a satisfacer las necesidades individuales, familiares, regionales y nacionales con una visión social y ética (Carreño et al., 2007). 
Tabla II. Niveles de Dominio Competencia Genérica Responsabilidad Social (UFRO).

\begin{tabular}{|c|c|c|}
\hline Nivel & Nivel de Dominio & Indicador conductual \\
\hline 1 & $\begin{array}{l}\text { Evidencia preocupación y r espeto con el } \\
\text { bienestar personal y del grupo. }\end{array}$ & $\begin{array}{l}\text { A.- Es responsable con los compromisos asumidos. } \\
\text { B.- Respeta normas y pautas socialmente consensuadas. } \\
\text { C.- Identifica y demuestra interés frente a problemas de } \\
\text { carácter social. }\end{array}$ \\
\hline 2 & $\begin{array}{l}\text { Asume las repercusiones y las } \\
\text { consecuencias de sus actos an ivel } \\
\text { personal, grupal y social. }\end{array}$ & $\begin{array}{l}\text { A.- Se interesa por el cuidado de sí mismo y manifiesta } \\
\text { conciencia respecto a cómo su accionar afecta a los otros. } \\
\text { B.- Posee un trato respetuoso hacia sus pares, profesores y } \\
\text { otras personas. } \\
\text { C.- Es proactivo y flexible en el análisis de soluciones de } \\
\text { problemas de carácter social. }\end{array}$ \\
\hline 3 & $\begin{array}{l}\text { Evalúa el impacto de sus acciones y } \\
\text { decisiones sobre el grupo ys u medio } \\
\text { ambiente, intentando influir sobre ellas de } \\
\text { manera activa. }\end{array}$ & $\begin{array}{l}\text { A.- Es consciente de que sus acciones repercuten en los } \\
\text { B.- Se preocupa porque sus acciones promuevan el bien } \\
\text { común en su entorno cercano. } \\
\text { C.- Analiza el impacto que tiene el conocimiento y la } \\
\text { práctica de su futura profesión en satisfacer las necesidades } \\
\text { de la sociedad. }\end{array}$ \\
\hline
\end{tabular}

Nivel 1: Competencia en la realización de una gama acotada de actividades, en su mayoría sencillas y de resultados predecibles; Nivel 2: Competencia en la realización de una gama mayor de actividades. El estudiante cuenta con un mínimo de autonomía, recibe un alto grado de supervisión y se desenvuelve de forma suficiente.

Nivel 3: Competencia en la realización de una gama variada de actividades, en diversos contextos. El estudiante actúa con mayor autonomía y asume la responsabilidad de su desempeño.

En ciencias forenses, más allá de la evidente necesidad de comunicación con los denominados "operadores jurídicos" (lo que obliga al perito a conocer profundamente las normas legales), o al desarrollo de sus tres pilares fundamentales de servicio -la actividad pericial, docente e investigadora-, la contribución sanitaria y social de la práctica forense actual suele pasar desapercibida en un contexto que está claramente necesitado de ella. La investigación en patología forense ofrece información útil y fiable en estudios sobre factores de riesgo y prevención en diversas áreas de la medicina como es el caso de la mortalidad por causas externas, infecciones o enfermedades hereditarias. Los datos obtenidos en las autopsias y la incorporación de estos al ámbito clínico ha logrado mejorar sustancialmente las estadísticas de mortalidad, no sólo en casos de muertes violentas (accidentes de tráfico, suicidios o reacciones adversas al consumo de tóxicos), sino también en aquellos que aun siendo naturales, permiten elaborar políticas de prevención en la población general y, de forma particular, en los familiares de los fallecidos por muerte súbita de origen cardiovascular (Lucena, 2014).

En lo estrictamente atingente a la odontología forense, Avon (2004) ha referido que todo profesional odontólogo posee la responsabilidad implícita de comprender las implicaciones jurídicas de su profesión: mantener registros legibles y legalmente acep- tables de sus pacientes, ayudar en la identificación de víctimas o de sospechosos criminales cuando es solicitado, o denunciar sospechas de abuso infantil cuando se ve confrontado con inusuales lesiones orales, representan profundos compromisos sociales ineludibles para cualquier graduado.

Responsabilidad Social es una CG distintiva para la Universidad de La Frontera, producto de la relevancia que se le confiere en la Misión, Plan de Desarrollo Estratégico y Política de Formación Profesional de esta casa de estudios (CIP).

\section{DISCUSIÓN}

La NAS, ha definido a la Odontología Forense como "la aplicación de la ciencia de la odontología al campo de la ley, focalizándose en diferentes áreas como son: la identificación de restos, la comparación de huellas de mordedura, la interpretación de lesiones orales y la malpraxis dental" (Committee on Identifying the Needs of the Forensic Science Community et al.). Sin lugar a dudas, esta es una concepción más amplia y contenedora que la referida a la sola identificación, y sobre todo, clarificadora de competencias y roles a asumir por el experto odontólogo en un equipo interdisciplinario. Sin embargo, este y otros conceptos fueron vertidos por la 
NAS bajo el sugerente título de "Fortaleciendo a las Ciencias Forenses en los Estados Unidos: Una manera de avanzar" (Strengthening Forensic Science in the United States: A Path Forward), informe de 254 páginas presentado por un Comité específico luego de una minuciosa investigación sobre las ciencias forenses identificadoras entre las que se incluyó al análisis de huellas de mordedura, una de las pericias más controvertidas (Fonseca et al., 2013). Este documento apuntó a resolver algunas de las necesidades emergentes en la comunidad científica forense, entre las que "aumentar el número de peritos calificados" y "promover el empleo de las mejores prácticas de recolección y análisis de evidencia forense asegurando calidad y consistencia tanto en técnicas como en tecnologías de uso forense para resolver crímenes, investigar muertes y proteger al público", resultaron ser críticas. Buscando consolidar un papel educador en este sistema, la NAS fue concluyente al referir que "...para corregir algunas de las deficiencias existentes, el punto de partida debería ser mejorar los programas de pre y postgrado, así como incrementar las oportunidades para la educación continua. La legitimación de las prácticas en las disciplinas científicas forenses debe basarse en conocimientos científicos, principios y prácticas validadas, lo que se aprende mejor a través de la educación formal, la capacitación y el desarrollo adecuado de la investigación" (Committee on Identifying the Needs of the Forensic Science Community et al.).

La ausencia real de autores de habla hispana en la literatura odontológica forense más actual y válida, originando nuevos conocimientos u homologando los ya reconocidos, contradice sustancialmente la tendencia global de exigir peritos actualizados, con opiniones fundadas en principios validados e imparciales, en pocas palabras, expertos con bases científicas (Fonseca). El prestigioso odontólogo forense $\mathrm{C}$. Michael Bowers refiere sobre el análisis de las huellas de mordedura que la bibliografía es "sorprendentemente escasa y carente de rigor científico" agregando que "la comunidad científica odontológica forense se ha mantenido silenciosa" sobre las condenas erróneas apoyadas en estas pericias (Fonseca et al.). Precisamente incluyendo a esta pericia, la NAS sostiene que la corrección de estas deficiencias podrá ser efectiva sólo si se mejoran los programas de pre y posgrado en ciencias forenses (Committee on Identifying the Needs of the Forensic Science Community et al.)
Chile. En Chile, la Odontología Legal y Forense no forma parte de los contenidos en educación superior según informan González et al. (2005). De acuerdo a los autores, y sobre un análisis de programas de estudio de 10 Facultades chilenas de Medicina, sólo 2 incorporan estos contenidos en sus currículos; "odontología forense" es el tema menos abordado (10\%); y sólo 2 publicaciones $(3,85 \%)$ fueron detectadas sobre esa disciplina. Aunque esta situación ha mostrado algunos cambios, donde algunas universidades han integrado esta disciplina en su formación de pregrado como asignaturas obligatorias o electivas (principalmente de manera teórica), no es una práctica generalizada, y carecen de una estandarización o regulación. Siendo Chile un país proclive a los desastres naturales como lo atestiguan sucesos de público conocimiento (terremotos, tsunamis, aluviones, erupciones volcánicas, etc.), o con un reconocido historial de personas detenidas/ desaparecidas durante el gobierno militar con inicio en 1973 y posteriores (además de actuales) necesidades de identificación, resulta particularmente curioso que las rutinas en odontología forense sean mínimas y básicas (Moreno, 2010), que existan sólo 10 odontólogos contratados por el Servicio Médico Legal (8 de ellos en Región Metropolitana) (www.sml.cl), el Registro Nacional de Prestadores Individuales de Salud acuse oficialmente nada más que 6 especialistas odontólogos legistas en todo el territorio (www.superdesalud.gob.cl), y que mientras en 2015 se inició un primer Programa de Especialización en Odontología Legal y Forense, a la fecha de esta publicación, aún no existe una cohorte local de especialistas en todo ese país.

Argentina. En Argentina, de las 3 carreras de especialización en Odontología Legal acreditadas por la Comisión Nacional de Educación -CONEAU- según su sitio oficial (www.coneau.edu.ar), dos de ellas se encuentran discontinuadas hace algunos años, y sus programas poseen una neta y casi total inclinación a los contenidos derivados del ejercicio de la profesión u odontología legal propiamente dicha -según los lineamientos de Moya Pueyo et al. (1994). Esto deja al recién titulado especialista, literalmente a su suerte para complementar de manera casi autodidacta su capacitación en la contraparte forense (Fonseca). Dado que existe una virtual ausencia de autores/publicaciones de esa nacionalidad en revistas arbitradas validadas, y de que tampoco existen datos en la literatura que refieran contenidos y estrategias de enseñanza-aprendizaje de programas de pregrado en la disciplina, se propone un abordaje 
de estos tópicos cuyos resultados serán sin dudas de gran relevancia para la odontología legal y forense argentina.

México. En México, los requerimientos de un apropiado examen, manejo, evaluación y presentación en juicio de las evidencias dentales, todos subrayados ya por Keiser-Nielsen (1980), obligan a una detenida reflexión de los elementos implicados por esta concepción en la formación del profesional odontólogo, de la visión integral de su perfil de egreso y de la adecuada formación basada en CGs así como en su Aprendizaje Basado en Problemas (ABP). Una revisión de los planes de estudios de los diez programas considerados en el ranking de las mejores universidades mexicanas (http:// mejoresuniversidadesdemexico.mx) demuestra que sólo en una universidad la asignatura es parte obligatoria de la plantilla de pregrado, en tres es optativa, en dos no se encuentra explícita y en cuatro directamente no es oferta académica. Evidentemente existe una pobre planeación en las organizaciones académicas internas, con la gestación de modelos de enseñanza que forman profesionales poco competentes para dar respuesta al problema de salud-enfermedad (López-Cámara \& Lara-Flores, 2006), menos aún aplicable a el modelo que se pondera. La tendencia actual hacia una enseñanza basada en CGs sorprendentemente no encuentran lugar en el escenario de la odontología legal y forense mexicana, lo que deriva en una práctica educativa que sólo alcanza niveles informativos creando un gran vacío motivacional y de aprendizaje. El panorama a nivel de postgrado se muestra débil y poco estudiado tanto en su calidad como en el impacto de los diplomados (la mayoría) o maestrías en la disciplina. De igual manera, y coincidiendo con lo que sucede a un nivel global, problemas como la violencia doméstica o el abuso infantil, son escenarios usualmente abarcados por disciplinas ajenas a la odontología legal y forense (Patel et al., 2014).

Siendo uno de los países con más tradición en la disciplina, y viviendo desde hace casi una década con niveles descomunales de violencia y muerte (Espinal-Enríquez \& Larralde, 2015), es curioso que la enseñanza de la odontología legal y forense en México se encuentre en una grave crisis existencialcognitiva, circunstancia que no refiere sólo a una realidad latinoamericana como se verá más adelante.

España. En España, la odontología legal y forense aun no está reconocida como especialidad, y su en- señanza solo aparece contemplada en los currículos de pregrado en algunas facultades de odontología. En estos pocos casos, como ocurre en Sevilla, sus contenidos se encuentran vertidos en una asignatura cuatrimestral en el cuarto curso del grado con la denominación "Profesionalismo y odontología legal y forense" (http://www.us.es). En el caso del postgrado, la oferta formativa en la disciplina es bastante limitada, los cursos de experto y máster universitario en odontología legal y forense han tenido escasa continuidad y algunos ya han dejado de impartirse. Actualmente existen algunas actividades de capacitación focalizadas sólo en la odontología legal (no así la forense) como es el curso de "Experto en Peritaciones en Odontología Legal y Forense, Valoración del daño dento-facial", en la Universidad Alfonso X de Madrid (http://www.uax.es) y los que organiza la Escuela de Medicina Legal adscrita a la Universidad Complutense de Madrid (http:// www.ucm.es).

Aunque la odontología es una pieza clave de la actividad forense, paradójicamente los odontólogos no están contemplados como candidatos para ocupar un puesto en los Institutos de Medicina Legal españoles. En la relación de puestos de trabajo de los mismos no aparece recogida la figura del odontólogo forense y, por lo tanto, no poseen plazas de acceso a estos servicios. Existen los mecanismos legales para que un odontólogo forense pueda ser llamado como asesor externo, pero esta posibilidad es raramente utilizada.

Esta realidad contrasta con el interés cada vez más creciente de los odontólogos por la disciplina forense. Como ejemplo, basta señalar el curso teórico-práctico "Análisis de huellas de mordeduras" celebrado en Écija (España) en 2013 y 2014, organizado por la Sociedad Española de Patología Forense -SEPAF-, con la colaboración de la Asociación Española de Antropología y Odontología Forense AEAOF- y la Sociedad de Odontoestomatólogos Forenses Iberoamericanos-SOFIA-. El curso contó con el aval científico del Consejo General de Dentistas de España y tuvo una demanda sin precedentes con la asistencia de odontólogos, médicos forenses y antropólogos de toda España, Italia, Venezuela y Argentina (Maxillaris, Comunicación Global en Odontología, 2014).

La situación en Europa, como se mencionaba anteriormente, es bastante similar a la realidad Española y no existen requerimientos especiales para 
llegar a ser odontólogo forense, al contrario de lo que ocurre en otros países del mundo (Estados Unidos, Australia, Sudáfrica). Algunas universidades Francesas ofrecen numerosos cursos de postgrado en odontología forense (Nantes, Nancy, Montpelier, Toulouse, Marsella y Poitiers). Solo hay dos universidades que ofrecen títulos de master en odontología forense; el de la KU de Leuven (Bélgica) (http:// www.kuleuven.be) y el de la Universidad de Glamorgan (Reino Unido) (http://courses.glam.ac.uk). El primero tiene una duración de un año a tiempo completo o dos años a tiempo parcial y se desarrolla en inglés. Debido a la escasez de programas y cursos en el período de postgrado, algunos autores consideran que es necesario un impulso general para mejorar y extender los programas de odontología forense en el pregrado (Lorkiewicz-Muszynska et al., 2013).

Wadhwan et al. (2014), afirman que en India, donde la gran mayoría de los odontólogos desconocen las pocas oportunidades existentes para una capacitación formal en el área, no existen laboratorios específicos, y la misma disciplina no ha sido incluida en los currículos académicos sino hasta hace poco tiempo. Mientras que la mejor instancia para recabar información continúa siendo la publicación, son muy pocos los odontólogos interesados en leerlas. Los autores proponen revertir esta situación de inadecuado conocimiento, pobre actitud y falta de concientización a través de la inclusión efectiva de la Odontología Forense en el currículo de pre y posgrado. Estados Unidos inició la educación de pregrado en odontología forense en los años '60 y el Reino Unido en los '70, y el prestigioso Gosta Gustafson subrayó la necesidad de que la disciplina fuera parte básica de estos programas (Singh et al., 2013). Aunque con parciales resultados positivos reportados por diversos autores (Avon; Syrjänen \& Sainio, 1990), otros han postulado que la falta de crecimiento de la educación forense en las facultades de odontología puede atribuirse a la falta de instructores calificados, falta de interés en las instituciones para desarrollarlos o falta de tiempo para realizar otra cosa que no sea "mostrar y hablar" (Hermsen \& Johnson, 2012). En definitiva, hay carencia de instituciones comprometidas y apropiadas estrategias para el aprendizaje significativo, este último la razón fundamental de un apropiado proceso de enseñanza-aprendizaje en el interior del aula (Huitzil Muñoz).

Los estudiantes necesitan ayuda para asumir e incrementar responsabilidad por el desarrollo de sus propias habilidades. Esto requiere presentarles opor- tunidades curriculares para desplegar CGs más transparentes y explícitas, a la vez ofrecidas por educadores igualmente entrenados y comprometidos (MurdochEaton \& Whittle). Apuntalando una Odontología Basada en Evidencia, debe considerarse que el estudiante necesita desarrollar su Pensamiento Crítico a la hora de evaluar no sólo la literatura existente sino también los mejores procedimientos disponibles (Lallier). La odontología forense debería ser parte integral del currículo en una facultad de odontología, pues ofrece la oportunidad de una experiencia activa de aprendizaje, idear desafíos, desarrollar el pensamiento crítico y aplicar el método científico (Stoeckel et al., 2007). Los resonantes casos de pericias odontológicas forenses con resultados cuestionados e insuficiencia científica en sus bases (Fonseca et al.), podrían haber tenido otra suerte de haber reconocido honestamente los límites de informar más allá de lo posible. La habilidad del Pensamiento Crítico ofrece un mecanismo de prevención de la "malpraxis del perito" (Fonseca); la honestidad no sólo se transforma en una cualidad deseable para el experto forense sino que posibilita también esclarecer su rol según la CG Responsabilidad Social: los fríos números de falsos positivos y falsos negativos pasan a transformarse en personas inocentes encarceladas, o culpables exonerados respectivamente.

La rápida evolución de las necesidades de la sociedad requiere profesionales globalmente competentes capaces de aplicar conocimientos y desplegar habilidades hoy completamente invisibles y desconocidas, más aún en la Odontología Forense. La Facultad de Odontología de la Universidad de La Frontera desarrolla actualmente su primera cohorte en Odontología Legal y Forense como ramo electivo de pregrado, basándose en las CGs Pensamiento Crítico y Responsabilidad Social. Esta propuesta ha pretendido implementar las necesarias estrategias catalizadoras de destrezas para contribuir a un aprendizaje significativo a lo largo de la vida, habilidades para autorregularse y adaptarse a cambios en las ideas y en el contexto en que se mueven (Murdoch-Eaton \& Whittle). La nociva "autosuficiencia académica" descrita por Jones, ha sido controlada con la inclusión de educadores de otras disciplinas en su programa de estudio (Figs. 1 y 2), modelo que permite inyectar más amplitud y perspectiva crítica.

No se trata sólo de capacitar eventuales peritos, se trata de vincularlos con el medio ofreciéndoles las herramientas necesarias para, "desde la muerte... alegrarse de estar ayudando a la vida" (Lucena). 


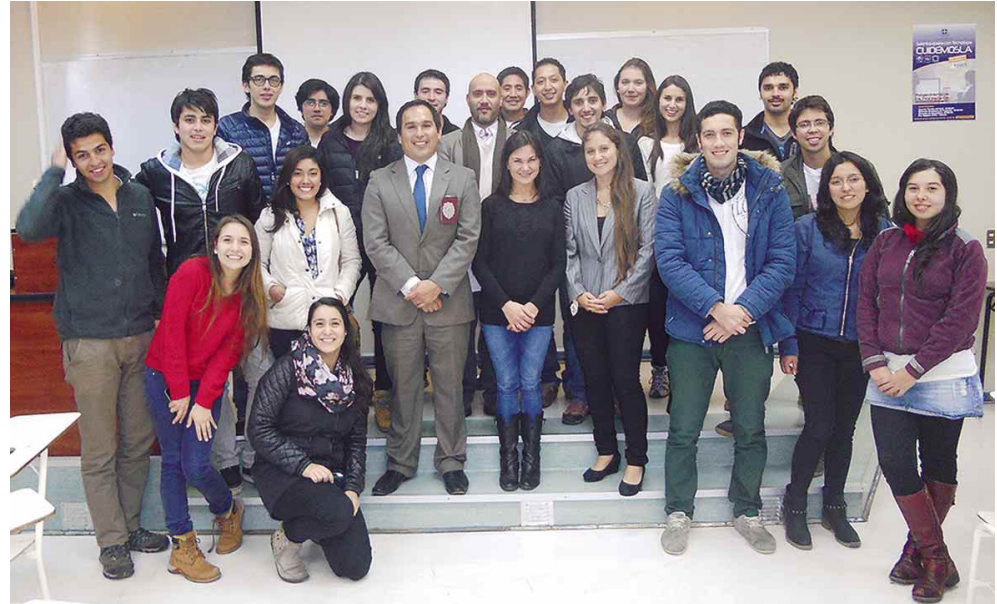

Fig. 1. Primera cohorte 2015, asignatura electiva Odontología Legal y Forense, Escuela de Odontología, Facultad de Odontología, Universidad de La Frontera -UFRO-. Seminario sobre el tema "Abuso Infantil" impartido por personal de la Brigada de Delitos Sexuales y Menores BRISEXME- de la Policía de Investigaciones, Temuco y docentes de la asignatura Clínica Odontológica Integral Pediátrica de esa casa de estudios. Se abordaron los temas: signos orales de abuso y maltrato infantil, legislación vigente, obligación del cirujano dentista y protocolo de denuncia, presentación de la BRISEXME, estadísticas regionales y análisis de casos (Actividad efectiva según programación de la asignatura, abril de 2015).

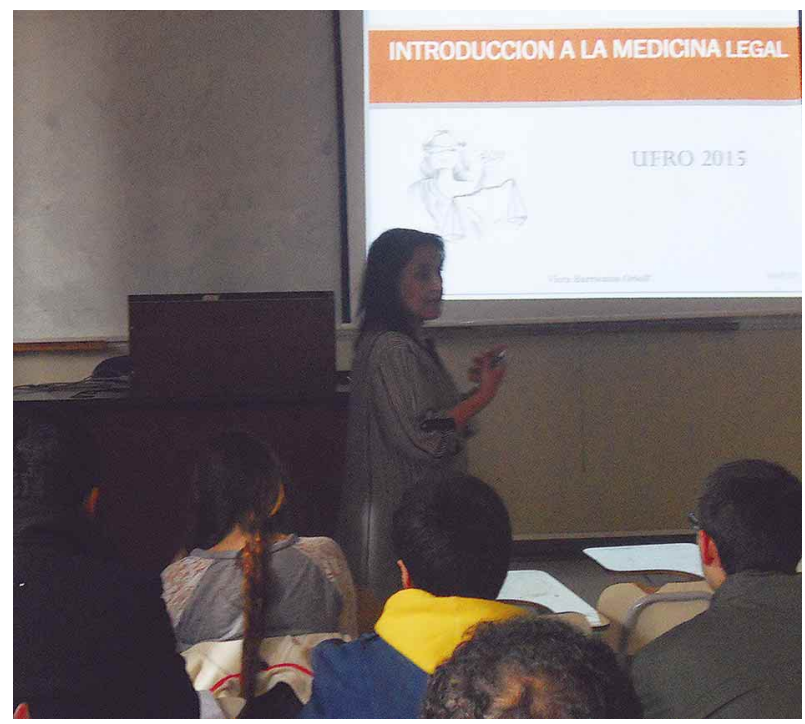

Fig. 2. Primera cohorte 2015, asignatura electiva Odontología Legal y Forense, Escuela de Odontología, Facultad de Odontología, Universidad de La Frontera -UFRO-. Seminario sobre el tema "Lesionología y Autopsia" impartido por la Prof. Dra. Viera Barrientos Orloff, Directora Regional del Servicio Médico Legal -SML-, Región de la Araucanía. Se abordaron los temas: introducción a la medicina legal, presentación del SML Regional, valoración de lesiones, legislación vigente, autopsia medicolegal, inserción del cirujano dentista en procesos de identificación y análisis de casos (Actividad efectiva según programación de la asignatura, mayo de 2015).
ORTIZ, J.; FONSECA, G. M.; CANTÍN, M.; HUITZIL, E. \& LUCENA, J. Generic skills for education in forensic dentistry: Critical thinking and social responsibility. Int. J. Odontostomat., 9(2):263-272, 2015.

ABSTRACT: The competence-based training nowadays represents a strategy of teaching-learning, which supports the student to prepare him/her for better performance in the increasingly complex social environments through the development of their own skills. This model requires that educational institutions must offer curricular opportunities to develop explicit and transparent generic skills (GSs). The contextual challenges of forensic sciences in general, and of the legal and forensic dentistry in particular, have to determine a necessary adaptation of their conceptual, procedural and attitudinal contents to define their specific fields, holistic development of their areas of knowledge, the acquisition of skills and experience, orientation toward professional ethical values, and integration of all these contents. We present Critical Thinking and Social Responsibility as the more suitable GSs for a training focused on the legal and forensic dentistry. To take up the challenge to improve the quality, consistency and adequacy of not only of new future experts but also of the professional healthcare practitioner, and the significance of formalizing these aspects with the realities and prevailing paradigms today in some Spanish-speaking countries is discussed.

KEY WORDS: forensic dentistry, generic skills, teaching-learning process.

\section{REFERENCIAS BIBLIOGRÁFICAS}

Avon, S. L. Forensic odontology: the roles and responsibilities of the dentist. J. Can. Dent. Assoc., 70(7):453-8, 2004.

Bergsmann, E.; Schultes, M. T.; Winter, P.; Schober, B. \& Spiel, C. Evaluation of competence-based teaching in higher education: From theory to practice. Eval. Program Plann., 52(1):1-9, 2015.

Carreño, P.; Bonilla, S.; Rubio, C.; Cortés, M. \& Ojeda, J. Responsabilidad social en la formación del químico farmacéutico de la Universidad de Valparaíso, Chile. Edusfarm, Rev. Educ. Sup. Farm., (2):1-12, 2007.

Ciocca, G. L. Odontología Médico-Legal. Aspectos Forenses, Profesionales y Sociales. Santiago, Ed. Jurídicas de Santiago, 2010. 
Committee on Identifying the Needs of the Forensic Science Community; National Research Council (US) Committee on Science Technology and Law \& National Research Council (US) Committee on Applied and Theoretical Statistics. Strengthening forensic science in the United States : a path forward. Committee on Identifying the Needs of the Forensic Science Community; Committee on Science, Technology \& Law Policy and Global Affairs, Committee on Applied and Theoretical Statistics, Division on Engineering and Physical Sciences. National Research Council of the National Academies. Washington D. C., National Academies Press, 2009.

El Tantawi, M. M.; Abdelaziz, H.; AbdelRaheem, A. \& Mahrous, A. Using peer-assisted learning and role-playing to teach generic skills to dental students: the health care simulation model. $J$. Dent. Educ., 78(1):85-97, 2014.

Espinal-Enríquez, J. \& Larralde, H. Analysis of México's NarcoWar Network (2007-2011). PLoS One, 10(5):e0126503, 2015.

Fonseca, G. M. Odontología Forense: La Calificación del Perito. Rev. La Peritia, 4(1):21-4, 2012.

Fonseca, G. M.; Briem-Stamm, A. D.; Cantín, M.; Lucena, J. \& Bentkovski, A. Odontología Forense I: Las Huellas de Mordedura. Int. J. Odontostomat., 7(1):149-57, 2013.

González, L.; Inzunza, J. A.; Bustos, L.; Vallejos, C. \& Gutiérrez, R. Docencia e investigación en Medicina Legal: Situación actual y desafíos para las Facultades de Medicina de Chile. Rev. Med. Chil., 133(7):805-12, 2005.

Hermsen, K. P. \& Johnson, J. D. A model for forensic dental education in the predoctoral dental school curriculum. J. Dent. Educ., 76(5):553-61, 2012.

Huitzil Muñoz, E. E. Odontología Forense: Perspectivas Educacionales. F. O. P. J., 2(4):13-5, 2011.

Jones, A. Redisciplining generic attributes: the disciplinary context in focus. Stud. High. Educ., 34(1):85-100, 2009.

Keiser-Nielsen, S. Person Identification by Means of the Teeth: A Practical Guide. Bristol, John Wright \& Sons Ltd., 1980.

Lallier, T. E. Introducing evidence-based dentistry to dental students using histology. J. Dent. Educ., 78(3):380-8, 2014.

Lucena, J. Ser médico....y patólogo forense. Rev. Clin. Esp., 214(4):224-6, 2014.

López-Cámara, V. \& Lara-Flores, N. L. Enseñanza, investigación y práctica profesional de la odontología mexicana en el año 2003. Rev. Cienc. Clín., 7(1):23-9, 2006.

Lorkiewicz-Muszynska, D.; Przystanska, A.; Labecka, M. \& Kruszelnicki A. Current Status of Forensic Odontology Education - the Underestimation of Needs? Dent. Med. Probl., 50(2):217-22, 2013.

Mason, T. \& Phipps, D. Forensic learning disability nursing skills and competencies: a study of forensic and non-forensic nurses. Issues Ment. Health Nurs., 31(11):708-15, 2010.
Maxillaris, Comunicación Global en Odontología. Dientes, testigos de la vida y la muerte, 2014. Disponible en: https:// www.maxillaris.com/maximas-20140130-Dientes-testigos-dela-vida-y-la-muerte.aspx

Moreno, R. Procedimientos Médico Forenses en respuesta al Terremoto y Tsunami en Constitución (Chile), Febrero y Marzo de 2010. F. O. P. J., 1(2):20-6, 2010.

Moya Pueyo, V.; Roldán Garrido, B. \& Sánchez Sánchez, J. A. Odontología Legal y Forense. Barcelona, Masson, 1994.

Murdoch-Eaton, D. \& Whittle, S. Generic skills in medical education: developing the tools for successful lifelong learning. Med. Educ., 46(1):120-8, 2012.

Patel, N.; Bailey, E.; Mahdmina, A.; Lomax, A. \& Coulthard, P. Domestic violence education for UK and Ireland undergraduate dental students: a five-year perspective. J. Dent. Educ., 78(8):1162-6, 2014.

Singh, N.; Grover, S.; Kaur, G. \& Sahuwala, A. Forensic odontology: Educating the dental students. I. J. O. H. R. R.,1(2):7-11, 2013.

Singh, N. N.; Gowhar, O.; Ain, T. S. \& Sultan, S. Exploring trends in forensic odontology. J. Clin. Diagn. Res., 8(12):ZC28-30, 2014.

Stoeckel, D. C.; Merkley, P. J. \& McGivney, J. Forensic dental training in the dental school curriculum. J. Forensic Sci., 52(3):684-6, 2007.

Syrjänen, S. M. \& Sainio, P. Forensic dentistry--recent development towards an independent discipline in modern dentistry. Proc. Finn. Dent. Soc., 86(3-4):157-70, 1990.

Centro de Innovación Profesional (CIP). Diccionario de Competencias Genéricas. Temuco, Universidad de La Frontera, 2015. Disponible en: http://competenciasgenericas.ufro.cl/index.php/ diccionario

Wadhwan, V.; Shetty, D. C.; Jain, A.; Khanna, K. S. \& Gupta, A. A call for a new speciality: Forensic odontology as a subject. $J$. Forensic Dent. Sci., 6(2):97-100, 2014.

Wesselink, R.; Dekker-Groen, A.; Biemans, H. \& Mulder, M. Using an instrument to analyse competence-based study programmes: experiences of teachers in Dutch vocational education and training. J. Curric. Stud., 42(6):813-29, 2010.

Dirección para Correspondencia:

Gabriel M. Fonseca, DDS, Ph.D.

Facultad de Odontología

Universidad de La Frontera

Avenida Francisco Salazar 01145

Temuco

CHILE

Email: gabriel.fonseca@ufrontera.cl

Recibido : 15-03-2015

Aceptado: 17-06-2015 